Annales Academiæ Scientiarum Fennicæ

Mathematica

Volumen 37, 2012, 191-201

\title{
THE MULTIPLE-SLIT VERSION OF LOEWNER'S DIFFERENTIAL EQUATION AND POINTWISE HÖLDER CONTINUITY OF DRIVING FUNCTIONS
}

\author{
Sebastian Schleissinger \\ University of Würzburg, Department of Mathematics \\ 97074 Würzburg, Germany; sebastian.schleissinger@mathematik.uni-wuerzburg.de
}

\begin{abstract}
We consider the chordal Loewner differential equation for multiple slits in the upper half-plane and relations between the pointwise Hölder continuity of the driving functions and the generated hulls. The first result generalizes a result of Lind that gives a sufficient condition for driving functions to generate simple curves. The second result translates the property that the hulls locally look like straight lines at their starting points into a condition for the driving functions.
\end{abstract}

\section{Introduction}

Suppose $\gamma:[0, E] \rightarrow \overline{\mathbf{H}}$ is a simple curve with

$$
\gamma(0) \in \mathbf{R} \quad \text { and } \quad \Gamma:=\gamma(0, E] \subset \mathbf{H}:=\{z \in \mathbf{C} \mid \operatorname{Im}(z)>0\} .
$$

For every $t \in[0, E]$ we can consider the slit half-plane $H_{t}:=\mathbf{H} \backslash \gamma[0, t]$ and the unique conformal map $g_{t}: H_{t} \rightarrow \mathbf{H}$ with $g_{t}(z)=z+\frac{b(t)}{z}+\mathcal{O}\left(|z|^{-2}\right)$ near infinity. By changing the parameterization of $\gamma$, we can achieve $b(t)=2 t$. Then $\gamma$ is said to be parameterized by half-plane capacity. In this case, $g_{t}(z)$ is the solution of the initial value problem

$$
\dot{g}_{t}(z)=\frac{2}{g_{t}(z)-U(t)}, \quad g_{0}(z)=z,
$$

where $U(t)$ is a continuous real-valued function with $U(t)=\lim _{z \rightarrow \gamma(t)} g_{t}(z)$.

$U(t)$ is called driving function of $\Gamma$ and it encodes all geometric and topological properties of $\Gamma$. On the other hand, given a continuous function $U:[0, E] \rightarrow \mathbf{R}$ and $z \in \mathbf{H}$, we can solve the initial value problem (1.1). $g_{t}(z)$ may not exist for all $t$, so we define $T_{z}$ as the supremum of all $t$ such that the solution exists up to time $t$ and $g_{t}(z) \in \mathbf{H}$. Let

$$
H_{t}:=\left\{z \in \mathbf{H} \mid T_{z}>t\right\} .
$$

Then $g_{t}$ is the unique conformal mapping from $H_{t}$ onto $\mathbf{H}$ with

$$
g_{t}(z)=z+\frac{2 t}{z}+\mathcal{O}\left(|z|^{-2}\right) \quad \text { for } z \rightarrow \infty .
$$

The mapping $t \mapsto H_{t}$ is continuous if we endow unbounded subdomains of $\mathbf{H}$ with the topology that is induced by the Carathéodory kernel convergence with respect to $\infty$, which will be denoted by $\stackrel{\text { Cara }}{\longrightarrow}$. If we define $K_{t}:=\mathbf{H} \backslash H_{t}$, then we obviously have $K_{t} \subset K_{s}$ for $t \leq s$, so that $\left\{K_{t}\right\}_{t \in[0, E]}$ is a family of growing subsets of the upper

doi:10.5186/aasfm.2012.3720

2010 Mathematics Subject Classification: Primary 30C20, 30C35.

Key words: Univalent function, Loewner equation, slit map, slit domain. 
half-plane. Generally, a bounded subset $A \subset \mathbf{H}$ with the property $A=\mathbf{H} \cap \bar{A}$ such that $\mathbf{H} \backslash A$ is simply connected will be called a (compact) hull.

If the hulls $K_{t}$ describe a curve $\gamma(t)$ that intersects itself, say at $t=E$, then $K_{E}$ will be "more" than just a curve: the set $\mathbf{H} \backslash \gamma[0, E]$ is not simply connected then and the domain $\mathbf{H} \backslash K_{E}$ will be the unbounded component of $\mathbf{H} \backslash \gamma[0, E]$. So one question nearly suggests itself:

When does $K_{t}$ describe a growing simple curve?

This general problem is not completely solved yet. However, there are partial results, like the connection of quasislits to Hölder continuous driving functions.

A slit in $\mathbf{H}$ is called quasislit if it is a a quasiarc that approaches $\mathbf{R}$ nontangentially, where a quasiarc is just the image of a line segment under a quasiconformal self-homeomorphism of the plane.

Let $\operatorname{Lip}\left(\frac{1}{2}\right)$ denote the set of all $\frac{1}{2}$-Hölder continuous functions $U:[0, E] \rightarrow \mathbf{R}$, i.e., there is a $c>0$ such that

$$
|U(t)-U(s)| \leq c \sqrt{|s-t|}, \quad s, t \in[0, E],
$$

and let $\|U\|_{\frac{1}{2}}$ be the smallest possible value for $c$.

Lind, Marshall and Rohde proved the following connection between $\operatorname{Lip}\left(\frac{1}{2}\right)$ and quasislits, see [MR05, Li05].

Theorem 1.1. If $K_{E}$ is a quasislit, then $U \in \operatorname{Lip}\left(\frac{1}{2}\right)$. Conversely, if $U \in \operatorname{Lip}\left(\frac{1}{2}\right)$ with $\|U\|_{\frac{1}{2}}<4$, then $K_{E}$ is a quasislit.

In the following we don't work with quasislits, but we take a look at the connection between the "left and right Hölder continuity" of the driving function and the generated hulls. We denote by $\operatorname{Lip}_{L}\left(\frac{1}{2}\right)$ the set of all "pointwise left $\frac{1}{2}$-Hölder continuous" functions, so that for every $t \in(0, E]$ there is a $c>0$ and an $\varepsilon>0$ such that

$$
|U(t)-U(s)| \leq c \sqrt{t-s} \text { for all } s \in[t-\varepsilon, t] .
$$

The results in [Li05] reveal that $U$ already generates a growing slit if we assume that $U \in \operatorname{Lip}_{L}\left(\frac{1}{2}\right)$ with

$$
\limsup _{h \downarrow 0} \frac{|U(s)-U(s-h)|}{\sqrt{h}}<4
$$

for every $s \in(0, E]$. The curve need not be a quasislit then, of course.

The first aim of the present paper is to extend Lind's result to the more general situation of the multiple slit version of Loewner's differential equation (or multifinger case). This equation is also used in physics in order to study several growth phenomena, such as viscous fingering in Hele-Shaw cells, see, e.g., [GS08].

Given continuous functions $\lambda_{j}, U_{j}:[0, E] \rightarrow \mathbf{R}, j=1, \ldots, n$ with $\lambda_{j}(t) \geq 0$ and $\sum_{j} \lambda_{j}(t) \equiv 1$, then the initial value problem of the $n$-slit version reads

$$
\dot{g}_{t}(z)=\sum_{j=1}^{n} \frac{2 \lambda_{j}(t)}{g_{t}(z)-U_{j}(t)}, \quad g_{0}(z)=z .
$$

This equation arises if we consider $n$ disjoint simple curves growing from the real line into the upper half-plane. The images of their tips at time $t$ under $g_{t}$ are exactly the values of the driving functions $U_{j}(t)$. Conversely, the solution of (1.3) always gives 
us conformal mappings $g_{t}: \mathbf{H} \backslash K_{t} \rightarrow \mathbf{H}$, where the compact hulls $K_{t}$ are defined as in the case of the one-slit version.

By using techniques of [Li05], we will prove the following statement which generalizes the sufficient condition (1.2).

Theorem 1.2. If $U_{j}(t)<U_{j+1}(t)$ for all $t$ and $j=1, \ldots, n-1$ and for every $j$ and $t \in(0, E]$ we have $U_{j} \in \operatorname{Lip}_{L}\left(\frac{1}{2}\right)$ with

$$
\limsup _{\varepsilon \downarrow 0} \frac{\left|U_{j}(t)-U_{j}(t-\varepsilon)\right|}{\sqrt{\varepsilon}}<4 \sqrt{\lambda_{j}(t)},
$$

then $K_{E}$ consists of $n$ disjoint simple curves.

Remark. Hence the "pointwise left $\frac{1}{2}$-Hölder continuous" condition (1.4) forces $K_{E}$ to fall into $n$ disjoint simple curves. The condition $U_{j}(t)<U_{j+1}(t)$ is necessary for this simple-curve problem, but one can also get simple curves by driving functions that do not satisfy (1.4). However, this condition is sharp in some sense, see Section 3.1 and Proposition 3.1.

As we shall now see, the "right Hölder continuity" of a driving function in $t=0$ determines the way how the connected components of the sets $K_{t}$ start from $U_{1}(0), \ldots, U_{n}(0)$ into the upper half-plane. In order to make this precise we introduce the following definition:

Let $\varphi \in(0, \pi)$. We say that $K_{t}$ approaches $\mathbf{R}$ at $U_{j}(0)$ in $\varphi$-direction if for every $\varepsilon>0$ there is a $t_{0}>0$ such that the connected component of $K_{t_{0}}$ having $U_{j}(0)$ as a boundary point is contained in the set $\left\{z \in \mathbf{H} \mid \varphi-\varepsilon<\arg \left(z-U_{j}(0)\right)<\varphi+\varepsilon\right\}$.

Theorem 1.3. Let $j \in\{1, \ldots, n\}$ and suppose $U_{j}(0) \neq U_{k}(0)$ for all $k \neq j$ and $\lambda_{j}(0) \neq 0$. The growing hulls $K_{t}$ approach $\mathbf{R}$ at $U_{j}(0)$ in $\varphi$-direction if and only if

$$
\lim _{h \downarrow 0} \frac{U_{j}(h)-U_{j}(0)}{\sqrt{h}}=\frac{2 \sqrt{\lambda_{j}(0)}(\pi-2 \varphi)}{\sqrt{\varphi(\pi-\varphi)}} .
$$

We can extend the definition of a straight line approach to $\mathbf{R}$ to a "sector approach": $K_{t}$ approaches $\mathbf{R}$ at $U_{j}(0)$ in a sector if there exist angles $\alpha, \beta \in(0, \pi)$ and a $t_{0}>0$ such that the connected component of $K_{t_{0}}$ near $U_{j}(0)$ is contained in

$$
\left\{z \in \mathbf{H} \mid \alpha<\arg \left(z-U_{j}(0)\right)<\beta\right\} .
$$

Now, Theorem 1.3 suggests the following question:

Do the growing hulls $K_{t}$ approach $\mathbf{R}$ at $U_{j}(0)$ in a sector if and only if

$$
\limsup _{h \downarrow 0} \frac{\left|U_{j}(h)-U_{j}(0)\right|}{\sqrt{h}}<\infty ?
$$

The remainder of the paper is organized as follows. In Section 2 we look at basic properties of the $n$-slit equation (1.1) and prove Theorem 1.3 by an application of the scaling property. Section 3 discusses the simple-curve problem for the multiple slit equation. We look at necessary conditions in 3.1 and the proof of Theorem 1.2 is given in Section 3.2, where it is split into three auxiliary lemmas.

Acknowledgement. The author wants to thank the anonymous referee for taking the time to evaluate the manuscript and providing constructive and valuable comments. 


\section{The scaling property and approach to $\mathrm{R}$}

For the rest of this paper, we will confine ourselves to the case $n=2$, in order to simplify notation. This case already carries the main difference between the one-slit and the multiple slit equation, namely the fact that $K_{t}$ need not be connected any longer.

In order to guarantee solutions of (1.3), we will assume that $\lambda_{1}, \lambda_{2}, U_{1}, U_{2}$ are continuous functions (see Theorem 4.6 in [La05]). Furthermore we require that the two coefficients $\lambda_{j}(t)$ are positive, i.e., we are dealing with the following initial value problem:

$$
\begin{aligned}
& \dot{g}_{t}(z)=\frac{2 \lambda_{1}(t)}{g_{t}(z)-U_{1}(t)}+\frac{2 \lambda_{2}(t)}{g_{t}(z)-U_{2}(t)}, \quad g_{0}(z)=z, \\
& \lambda_{1}(t), \lambda_{2}(t)>0, \quad \lambda_{1}(t)+\lambda_{2}(t)=1 \quad \text { for all } t \in[0, E] .
\end{aligned}
$$

Suppose the hull $K$ is generated by $U_{1}(t), U_{2}(t), \lambda_{1}(t), \lambda_{2}(t)$. We mention two simple operations on $K$ which can be translated into transformations of the driving functions by elementary calculations.

- Scaling: For $d>0, d K$ can be generated by $d U_{j}\left(t / d^{2}\right), \lambda_{j}\left(t / d^{2}\right), j=1,2$.

- Translation: For $d \in \mathbf{R}, K+d$ can be generated by $U_{j}(t)+d, \lambda_{j}(t), j=1,2$.

Example 2.1. Suppose the generated hulls $K_{t}$ consist of two different line segments starting in zero and assume that we have constant coefficients $\lambda_{j}$. Then the scaling property implies $U_{j}(t)=c_{j} \sqrt{t}$ with $c_{1}<c_{2}$, provided that $U_{1}$ belongs to the left segment. The values of the constant coefficients $\lambda_{j}$ stand for the sizes of the two segments relative to each other.

From now on we will assume that the time endpoint $E=1$, which is no loss of generality because of the scaling property.

All properties of the growing hulls that are invariant under scaling, e.g., the question whether they are slits or not, will lead to properties of $U_{j}$ that are invariant under the transformation $U_{j}(t) \mapsto \frac{1}{d} U_{j}\left(d^{2} t\right)$. That is why the $\frac{1}{2}$-Hölder continuity of driving functions enters the game.

Next we use the scaling property to prove Theorem 1.3.

Proof of Theorem 1.3 (with $n=2$ ). First we note that the following Loewner equation generates a straight line segment starting in 0 with angle $\varphi$ (see [La05], Example 4.12 and change the time $\left.t \mapsto \lambda_{2}(0) t\right)$ :

$$
\dot{g}_{t}(z)=\frac{2 \lambda_{2}(0)}{g_{t}(z)-c \sqrt{t}} \quad \text { with } c:=\frac{2 \sqrt{\lambda_{2}(0)}(\pi-2 \varphi)}{\sqrt{\varphi(\pi-\varphi)}} .
$$

We let $j=2$ and by translation we can assume that $U_{2}(0)=0$.

Now let $d>0$, then the corresponding conformal mappings $g_{t}(z, d)$ for the scaled hulls $d K_{t}$ satisfy

$$
\dot{g}_{t}(z, d)=\frac{2 \lambda_{1}\left(t / d^{2}\right)}{g_{t}(z, d)-d U_{1}\left(t / d^{2}\right)}+\frac{2 \lambda_{2}\left(t / d^{2}\right)}{g_{t}(z, d)-d U_{2}\left(t / d^{2}\right)}, \quad g_{0}(z, d)=z .
$$

If we choose $d$ large enough, the corresponding hull at $t=1$ will always have two connected components. Let $G_{d}$ be the one containing 0 . We will have to look at the limit case $d \rightarrow \infty$. 
First, let $h_{t}(z, d)$ be the solution of the Loewner equation

$$
\dot{h_{t}}(z, d)=\frac{2 \lambda_{2}(0)}{h_{t}(z, d)-d U_{2}\left(t / d^{2}\right)}, \quad h_{0}(z, d)=z,
$$

and let $H_{d}$ be the generated hull at $t=1$. Choose an $R>0$ and let $D_{R}:=\mathbf{H} \cap\{|z|<$ $R\}$. If we denote by $g(\cdot, d) \stackrel{\text { Cara }}{\longrightarrow} g$ the Carathéodory convergence for Loewner chains ${ }^{1}$, defined in [La05], p. 114, then we have

$$
g(\cdot, d) \stackrel{\text { Cara }}{\longrightarrow} g \text { for } d \rightarrow \infty \text { in } D_{R} \text { if and only if } h(\cdot, d) \stackrel{\text { Cara }}{\longrightarrow} g \text { for } d \rightarrow \infty \text { in } D_{R} .
$$

This can be shown by using the fact that left summand of (2.2) converges uniformly to 0 on $D_{R} \times[0,1]$ and that $\lambda_{2}\left(t / d^{2}\right)$ converges uniformly to $\lambda_{2}(0)$, again on $D_{R} \times[0,1]$.

Now, the hulls $K_{t}$ approach $\mathbf{R}$ at 0 in $\varphi$-direction if and only if $D_{R} \cap G_{d}$ converges to a line segment $L$ starting in 0 with angle $\varphi$ with respect to the Hausdorff topology ${ }^{2}$ for $d \rightarrow \infty$, which is, in this special case, equivalent to $\mathbf{H} \backslash G_{d} \stackrel{\text { Cara }}{\longrightarrow} \mathbf{H} \backslash L$. Because of the above relation of $g(\cdot, d)$ to $h(\cdot, d)$, this is equivalent to $H_{d} \rightarrow L$ or $\mathbf{H} \backslash H_{d} \stackrel{\text { Cara }}{\longrightarrow} \mathbf{H} \backslash L$.

We have to show that this corresponds to $U_{2}(t)=c \sqrt{t}+\mathcal{O}(\sqrt{t})$ for $t \downarrow 0$.

First, suppose $H_{d} \rightarrow L$. We can apply Theorem 4.3 from [LMR10] which implies $d U\left(t / d^{2}\right) \rightarrow c \sqrt{t}$ uniformly on [0,1], and therefore $U_{2}(t)=c \sqrt{t}+\mathcal{O}(\sqrt{t})$.

Conversely, if $U_{2}(t)=c \sqrt{t}+\mathcal{O}(\sqrt{t})$, then $d U\left(t / d^{2}\right) \rightarrow c \sqrt{t}$ uniformly. Uniform convergence of driving functions generally implies kernel convergence of the corresponding domains by Proposition 4.47 in [La05]. Consequently, $\mathbf{H} \backslash H_{d} \stackrel{\text { Cara }}{\longrightarrow} \mathbf{H} \backslash L$.

\section{The simple-curve problem}

Now we turn to the question:

When does equation (2.1) generate two disjoint simple curves?

First we derive two necessary conditions for this problem and in Section 3.2 we prove Theorem 1.2.

Again, we assume for the rest of this section that the time endpoint $E=1$ and that $\lambda_{1}(t), \lambda_{2}(t)>0$ for all $t \in(0,1]$. Note that this condition is automatically satisfied in the setting of Theorem 1.2.

3.1. Necessary conditions. If we knew that we had two curves with starting points $U_{1}(0)<U_{2}(0)$ and a time $t$ with $U_{1}(t)=U_{2}(t)$, then their two tips would coincide. Thus, as we require continuous driving functions, a necessary condition for having two curves is $U_{1}(t)<U_{2}(t)$ for all $t \in[0,1]$.

The following, so called backward Loewner equation is very useful for the simplecurve problem. For $T \in(0,1]$, we consider the initial value problem:

$$
\dot{f}_{t}(z)=\frac{-2 \lambda_{1}(T-t)}{f_{t}(z)-U_{1}(T-t)}+\frac{-2 \lambda_{2}(T-t)}{f_{t}(z)-U_{2}(T-t)}, \quad f_{0}(z)=z .
$$

$1 g(\cdot, d) \stackrel{\text { Cara }}{\longrightarrow} g(\cdot)$ if for every $\varepsilon>0$ and every $T \in[0,1], g_{t}(z, d)$ converges to $g_{t}(z)$ uniformly on $[0, T] \times\left\{z \in \mathbf{H} \mid \operatorname{dist}\left(z, K_{T}\right) \geq \varepsilon\right\}$.

${ }^{2}$ The Hausdorff distance between two compact subsets $A, B \subset \mathbf{C}$ is

$$
d_{H}(A, B)=\max \left\{\sup _{a \in A} \inf _{b \in B}|a-b|, \sup _{b \in B} \inf _{a \in A}|a-b|\right\} .
$$


$f_{t}(z)$ is defined for all $t \in[0, T]$ and $z \in \mathbf{H}$ and we have

$$
f_{T}(z)=g_{T}(z)^{-1} \text {. }
$$

The scaling and translation property also hold for the backward equation. Next we give another, non-trivial necessary condition.

Proposition 3.1. Let $U_{1}(t)<U_{2}(t)$ for all $t \in[0,1]$ and assume there is an $s \in(0,1]$ such that $\lambda_{2}(s)>0$ and

$$
\liminf _{h \downarrow 0} \frac{\left|U_{2}(s)-U_{2}(s-h)\right|}{\sqrt{h}} \geq 4 \sqrt{\lambda_{2}(s)} .
$$

Then $K_{s}$ does not consist of two disjoint simple curves.

Proof. Without loss of generality we assume that $s=1$ and $U_{2}(1)=0$. First, suppose that $\lim _{h \downarrow 0} U_{2}(1-h) / \sqrt{h}=4 \sqrt{\lambda_{2}(1)}$. Now consider the backward Loewner equation with $T=1$, scaled by $d>0$ :

$$
\dot{f}_{t}(z, d)=\frac{-2 \lambda_{1}\left(1-t / d^{2}\right)}{f_{t}(z, d)-d U_{1}\left(1-t / d^{2}\right)}+\frac{-2 \lambda_{2}\left(1-t / d^{2}\right)}{f_{t}(z, d)-d U_{2}\left(1-t / d^{2}\right)}, \quad f_{0}(z)=z .
$$

Let $d \rightarrow \infty$, as in the proof of Theorem 1.3 , then for every $t \in[0,1]$ the conformal mappings $f_{t}(z, d)$ converge in a neighborhood of 0 to the solution of

$$
\dot{f}_{t}(z)=\frac{-2 \lambda_{2}(1)}{f_{t}(z)-4 \sqrt{\lambda_{2}(1)} \sqrt{t}}, \quad f_{0}(z)=z,
$$

which does not generate a simple curve (see [LMR10], chapter 3 and change the time $\left.t \mapsto \lambda_{2}(1) t\right)$. But then, $f_{t}(z, d)$ is not a slit mapping for all $d>0$.

Now go back to the general case, where we have $\left|U_{2}(t)\right| \geq V(t)$ for all $t \in[0,1]$ and a continuous function $V$ with $\lim _{h \downarrow 0} V(1-t) / \sqrt{h}=4 \sqrt{\lambda_{2}(1)}$. Let us assume that $U_{2}(t) \geq V(t)$ for all $t \in[0,1]$. Next consider the real initial value problem

$$
\dot{y}(t)=\frac{2 \lambda_{1}(t)}{y(t)-U_{1}(t)}+\frac{2 \lambda_{2}(t)}{y(t)-V(t)}, \quad y\left(t_{0}\right)=y_{0} .
$$

For every $\varepsilon>0$ there are $t_{0}$ and $y_{0}<V\left(t_{0}\right)$ such that $-\varepsilon \leq y(1) \leq 0=V(1)$. The corresponding solution $x(t)$ of the original differential equation

$$
\dot{x}(t)=\frac{2 \lambda_{1}(t)}{x(t)-U_{1}(t)}+\frac{2 \lambda_{2}(t)}{x(t)-U_{2}(t)}, \quad x\left(t_{0}\right)=y_{0},
$$

satisfies $y(t) \leq x(t) \leq U_{2}(t)$. If $x(t)$ does not exist until $t=1$, we don't get simple curves. Otherwise we have $y(1) \leq x(1) \leq 0=U_{2}(1)$, so that these solutions also come arbitrarily close to 0 and we don't get simple curves in this case either.

Consequently, if $K_{1}$ consists of two disjoint simple curves, then, for every $s \in$ $(0,1]$, and $j \in\{1,2\}$, we have

$$
\begin{aligned}
& \limsup _{h \downarrow 0} \frac{\left|U_{j}(s)-U_{j}(s-h)\right|}{\sqrt{h}}<4 \sqrt{\lambda_{j}(s)} \quad \text { ("regular case"), or } \\
& \liminf _{h \downarrow 0} \frac{\left|U_{j}(s)-U_{j}(s-h)\right|}{\sqrt{h}}<4 \sqrt{\lambda_{j}(s)} \leq \limsup _{h \downarrow 0} \frac{\left|U_{j}(s)-U_{j}(s-h)\right|}{\sqrt{h}} \text { ("irregular case"). }
\end{aligned}
$$

So Theorem 1.2 states that the regular case is a sufficient condition for the simplecurve problem. 
3.2. Proof of Theorem 1.2. We begin with the following lemma.

Lemma 3.2. Let $\lambda>0,0 \leq \tau<2 \sqrt{\lambda}$ and $h_{n}$ be the following sequence of functions

$$
h_{1}(x)=x, \quad h_{n+1}(x)=x+\tau-\frac{4 \lambda}{h_{n}(x)} \quad \text { for } n \geq 1 .
$$

Let $x_{n}$ denote the largest zero of $h_{n}$. Then $\left(x_{n}\right)_{n}$ is an increasing sequence that converges to $4 \sqrt{\lambda}-\tau$. Furthermore, if $h_{n}(c) \geq 0$ for every $n \in \mathbf{N}$, then $c \geq 4 \sqrt{\lambda}-\tau$.

Proof. By induction, it can be shown that $h_{n+1}$ maps $\left(x_{n},+\infty\right)$ strictly monotonically onto $\mathbf{R}$. Consequently, $\left(x_{n}\right)_{n}$ is an increasing sequence. We prove that it is bounded above by $4 \sqrt{\lambda}-\tau$ by showing $h_{n}(4 \sqrt{\lambda}-\tau)>2 \sqrt{\lambda}$ for all $n \geq 1$ inductively: First,

$$
h_{1}(4 \sqrt{\lambda}-\tau)=4 \sqrt{\lambda}-\tau>4 \sqrt{\lambda}-2 \sqrt{\lambda}=2 \sqrt{\lambda}
$$

and for $n \geq 1$ we have

$$
h_{n+1}(4 \sqrt{\lambda}-\tau)=4 \sqrt{\lambda}-\tau+\tau-\frac{4 \lambda}{h_{n}(4 \sqrt{\lambda}-\tau)}>4 \sqrt{\lambda}-\frac{4 \lambda}{2 \sqrt{\lambda}}=2 \sqrt{\lambda} .
$$

Hence $x_{n}$ converges to

$$
\tilde{x} \leq 4 \sqrt{\lambda}-\tau .
$$

Obviously, we have $h_{n}(\tilde{x})>0$ for all $n$. Now suppose $h_{n}(\tilde{x}) \leq \sqrt{\lambda}$, then $h_{n+1}(\tilde{x})=$ $\tilde{x}+\tau-\frac{4 \lambda}{h_{n}(\tilde{x})} \leq \tilde{x}+\tau-4 \sqrt{\lambda} \leq 0$, a contradiction. Hence $h_{n}(\tilde{x})>\sqrt{\lambda}$. Furthermore, we get from $(3.1)$

$$
\begin{aligned}
h_{n}(\tilde{x})-h_{n+1}(\tilde{x}) & =h_{n}(\tilde{x})-\tilde{x}-\tau+\frac{4 \lambda}{h_{n}(\tilde{x})} \geq h_{n}(\tilde{x})-4 \sqrt{\lambda}+\frac{4 \lambda}{h_{n}(\tilde{x})} \\
& =\frac{h_{n}(\tilde{x})^{2}-4 \sqrt{\lambda} h_{n}(\tilde{x})+4 \lambda}{h_{n}(\tilde{x})}=\frac{\left(h_{n}(\tilde{x})-2 \sqrt{\lambda}\right)^{2}}{h_{n}(\tilde{x})} \geq 0 .
\end{aligned}
$$

It follows that the sequence $h_{n}(\tilde{x})$ is decreasing and bounded below by $\sqrt{\lambda}$. It converges to $\tilde{h}$ with

$$
\tilde{h}=\tilde{x}+\tau-\frac{4 \lambda}{\tilde{h}}
$$

So $\tilde{h}=\frac{\tilde{x}+\tau \pm \sqrt{(\tilde{x}+\tau)^{2}-16 \lambda}}{2}$ and hence $(\tilde{x}+\tau)^{2} \geq 16 \lambda$. As $\tilde{x}$ must be positive, we conclude $\tilde{x} \geq 4 \sqrt{\lambda}-\tau$ and together with (3.1) this implies $\tilde{x}=4 \sqrt{\lambda}-\tau$.

A great advantage of Lind's proof in [Li05] is the fact that we don't have to work with (2.1) for arbitrary initial values in the upper half-plane, but we can concentrate on the real initial value problem

$$
x\left(t_{0}\right)=x_{0}, \quad \dot{x}(t)=\frac{2 \lambda_{1}(t)}{x(t)-U_{1}(t)}+\frac{2 \lambda_{2}(t)}{x(t)-U_{2}(t)} .
$$

For the next lemma we define

$$
\lambda_{2}^{s}:=\min _{t \in[s, 1]} \lambda_{2}(t) .
$$


Lemma 3.3. Let $U_{2}(t) \in \operatorname{Lip}_{L}\left(\frac{1}{2}\right)$ with $U_{1}(t)<U_{2}(t)$. Suppose that $x(t)$ is a solution of (3.2) with $x_{0} \notin\left\{U_{1}\left(t_{0}\right), U_{2}\left(t_{0}\right)\right\}$ and $x(1)=U_{2}(1)$. Then

$$
\limsup _{h \downarrow 0} \frac{\left|U_{2}(1)-U_{2}(1-h)\right|}{\sqrt{h}} \geq 4 \sqrt{\lambda_{2}(1)} .
$$

Proof. We start with the case $U_{1}\left(t_{0}\right)<x_{0}<U_{2}\left(t_{0}\right)$, so that for all $t<1$ the solution satisfies $U_{1}(t)<x(t)<U_{2}(t)$. Let $T \in[0,1)$ be so close to 1 , that

- $x(t)-U_{1}(t)>\delta>\frac{1}{\lambda_{2}^{T}}\left(U_{2}(t)-x(t)\right)$ for a $\delta>0$ and all $t \in[T, 1]$ (which can be achieved because $\frac{1}{\lambda_{2}^{T}}$ is bounded and $U_{2}(t)-x(t)$ goes to zero when $t \rightarrow 1$ ) and

- $\left|U_{2}(1)-U_{2}(t)\right| \leq c \sqrt{1-t}$ for all $t \in[T, 1]$.

Next we define $\varepsilon:=\frac{2}{\delta} \sqrt{1-T}$ and we can assume (possibly by passing on to a larger $T)$

$$
\varepsilon<2 \sqrt{\lambda_{2}^{T}}
$$

Now $x(t)$ is decreasing in $[T, 1]$, since

$$
\dot{x}(t)=\frac{2 \lambda_{1}(t)}{x(t)-U_{1}(t)}+\frac{2 \lambda_{2}(t)}{x(t)-U_{2}(t)}<\frac{2}{\frac{1}{\lambda_{2}^{T}}\left(U_{2}(t)-x(t)\right)}+\frac{2 \lambda_{2}^{T}}{x(t)-U_{2}(t)}=0 .
$$

We will now show by induction that

$$
U_{2}(t)-x(t) \leq h_{n}(c) \sqrt{1-t} \text { for every } n \in \mathbf{N}, t \in[T, 1],
$$

where $h_{n}$ is the function from Lemma 3.2 with $\lambda=\lambda_{2}^{T}$ and $\tau=\varepsilon$. First we have

$$
U_{2}(t)-x(t) \leq U_{2}(t)-x(1)=U_{2}(t)-U_{2}(1) \leq c \sqrt{1-t}=h_{1}(c) \sqrt{1-t} .
$$

Now assume the inequality holds for one $n \in \mathbf{N}$. Then we have

$$
\dot{x}(t) \leq \frac{2}{\delta}+\frac{2 \lambda_{2}^{T}}{x(t)-U_{2}(t)} \leq \frac{2}{\delta}-\frac{2 \lambda_{2}^{T}}{h_{n}(c) \sqrt{1-t}} .
$$

Integrating yields

$$
x(1)-x(t) \leq \frac{2}{\delta}(1-t)-\frac{4 \lambda_{2}^{T}}{h_{n}(c)} \sqrt{1-t} \leq\left(\varepsilon-\frac{4 \lambda_{2}^{T}}{h_{n}(c)}\right) \sqrt{1-t} .
$$

This implies

$$
\begin{aligned}
U_{2}(t)-x(t) & \leq U_{2}(t)-U_{2}(1)+\left(\varepsilon-\frac{4 \lambda_{2}^{T}}{h_{n}(c)}\right) \sqrt{1-t} \\
& \leq\left(c+\varepsilon-\frac{4 \lambda_{2}^{T}}{h_{n}(c)}\right) \sqrt{1-t}=h_{n+1}(c) \sqrt{1-t}
\end{aligned}
$$

As $U_{2}(t)-x(t)$ is always positive, we conclude that $h_{n}(c) \geq 0$ for every $n \in \mathbf{N}$ and Lemma 3.2 tells us that

Finally, sending $T$ to 1 yields

$$
c \geq 4 \sqrt{\lambda_{2}^{T}}-\varepsilon
$$

$$
c \geq 4 \sqrt{\lambda_{2}^{1}}-0=4 \sqrt{\lambda_{2}(1)}
$$

The case $x_{0}>U_{2}\left(t_{0}\right)$ can be treated in the same way and in the case $x_{0}<U_{1}\left(t_{0}\right)$, the solution $x(t)$ cannot fulfill $x(1)=U_{2}(1)$. 
The point 1 in Lemma 3.3 can be replaced by any other time larger than zero and, of course, the result still holds if we switch the roles of $U_{1}$ and $U_{2}$. Thus, if we have $U_{1}, U_{2} \in \operatorname{Lip}_{L}\left(\frac{1}{2}\right)$ with $U_{1}(t)<U_{2}(t)$ and

$$
\limsup _{h \downarrow 0} \frac{\left|U_{j}(t)-U_{j}(t-h)\right|}{\sqrt{h}}<4 \sqrt{\lambda_{j}(t)}, \quad j=1,2,
$$

then the solution for any $x_{0} \notin\left\{U_{1}\left(t_{0}\right), U_{2}\left(t_{0}\right)\right\}$ will exist up to time $t=1$ and $x(1)$ cannot equal $U_{1}(1)$ or $U_{2}(1)$. In fact, there are even fixed intervals around $U_{1}(1)$ and $U_{2}(1)$ which cannot be reached by $x(1)$ for any initial value $x_{0}$.

Lemma 3.4. Let $U_{1}, U_{2} \in \operatorname{Lip}_{L}\left(\frac{1}{2}\right)$ with $U_{1}(t)<U_{2}(t)$ and

$$
\limsup _{h \downarrow 0} \frac{\left|U_{j}(t)-U_{j}(t-h)\right|}{\sqrt{h}}<4 \sqrt{\lambda_{j}(t)}
$$

for every $t \in(0,1]$ and $j=1,2$. Suppose that $x(t)$ is a solution of (3.2) with $x_{0} \notin\left\{U_{1}\left(t_{0}\right), U_{2}\left(t_{0}\right)\right\}$. Then there exists $\varepsilon>0$ such that

$$
\left|x(1)-U_{1}(1)\right|>\varepsilon \text { and }\left|x(1)-U_{2}(1)\right|>\varepsilon
$$

for every $x_{0} \notin\left\{U_{1}\left(t_{0}\right), U_{2}\left(t_{0}\right)\right\}$.

Proof. We prove the statement by contradiction, hence we assume that for every $\varepsilon>0$ there is an $x_{0}^{\varepsilon} \notin\left\{U_{1}\left(t_{0}\right), U_{2}\left(t_{0}\right)\right\}$ such that

$$
\left|x(1)-U_{1}(1)\right| \leq \varepsilon \quad \text { or } \quad\left|x(1)-U_{2}(1)\right| \leq \varepsilon .
$$

Without loss of generality we may assume that $U_{1}\left(t_{0}\right)<x_{0}^{\varepsilon}<U_{2}\left(t_{0}\right)$ and $U_{2}(1)-$ $x(1) \leq \varepsilon$. Now there is an $\epsilon>0$ such that for every $\varepsilon \in(0, \epsilon)$, the solution to the corresponding initial value $x_{0}^{\varepsilon}$ is decreasing in an interval $\left[T_{0}, 1\right]$ and $x(t)-U_{1}(t)>\delta$ for all $t \in\left[T_{0}, 1\right]$ and a $\delta>0$.

From now on we require $\varepsilon<\epsilon$ and furthermore we assume that $T \in[0,1)$ is so close to 1 that

- $T \geq T_{0}$,

- $\tau:=\frac{2}{\delta} \sqrt{1-T}<2 \sqrt{\lambda_{2}^{T}}$ and

- $\left|U_{2}(1)-U_{2}(t)\right| \leq c \sqrt{1-t}$ with $c<4 \sqrt{\lambda_{2}^{T}}-\tau$ for all $t \in[T, 1]$.

Again, we denote by $h_{n}$ the sequence

$$
h_{1}(x)=x, \quad h_{n+1}(x)=x+\tau-\frac{4 \lambda_{2}^{T}}{h_{n}(x)} \quad \text { for } n \geq 1 .
$$

Lemma (3.2) implies that there is an $N \in \mathbf{N}$ such that

$$
h_{N}(c)<0 .
$$

We take the smallest such $N$ and assume that $h_{n}(c)>0$ for all $n<N$. (If there is a $n$ with $h_{n}(c)=0$, one can pass on to a slightly greater $c$.) Next, define $e_{n}$ by

$$
\begin{aligned}
e_{1} & =\varepsilon, \\
e_{n+1} & =\varepsilon+\frac{4 \lambda_{2}^{T} e_{n}}{\left(h_{n}(c)\right)^{2}} \log \left(1+\frac{h_{n}(c)}{e_{n}}\right) \quad \text { for } 1 \leq n<N .
\end{aligned}
$$

Inductively one can easily show that for every $n \leq N$ we have $e_{n}>0$ and

$$
\lim _{\varepsilon \rightarrow 0} e_{n}=0 .
$$


Now we prove by induction that

$$
x(1)-x(t) \leq e_{n}-\varepsilon+\left(h_{n}(c)-c\right) \sqrt{1-t} \text { for all } t \in[T, 1] \text { and } n \leq N .
$$

The case $n=1$ states $x(1)-x(t) \leq 0$ which is true because $x(t)$ is decreasing. Next assume that the statement holds for a $n<N$. Then

$$
-x(t) \leq-x(1)-\varepsilon+e_{n}+\left(h_{n}(c)-c\right) \sqrt{1-t} \text { for all } t \in[T, 1] .
$$

Consequently,

$$
\begin{aligned}
U_{2}(t)-x(t) & \leq U_{2}(t)-U_{2}(1)+U_{2}(1)-x(1)-\varepsilon+e_{n}-\left(c-h_{n}(c)\right) \sqrt{1-t} \\
& \leq e_{n}+h_{n}(c) \sqrt{1-t} \text { for all } t \in[T, 1],
\end{aligned}
$$

which implies

$$
\dot{x}(t)=\frac{2 \lambda_{1}(t)}{x(t)-U_{1}(t)}+\frac{2 \lambda_{2}(t)}{x(t)-U_{2}(t)} \leq \frac{2}{\delta}-\frac{2 \lambda_{2}^{T}}{e_{n}+h_{n}(c) \sqrt{1-t}}
$$

and integrating gives

$$
\begin{aligned}
x(1)-x(t) & \leq \frac{2}{\delta}(1-t)-\frac{4 \lambda_{2}^{T}}{h_{n}(c)} \sqrt{1-t}+\frac{4 \lambda_{2}^{T} e_{n}}{\left(h_{n}(c)\right)^{2}} \log \left(1+\frac{h_{n}(c)}{e_{n}} \sqrt{1-t}\right) \\
& =\left(\frac{2}{\delta} \sqrt{1-t}-\frac{4 \lambda_{2}^{T}}{h_{n}(c)}\right) \sqrt{1-t}+\frac{4 \lambda_{2}^{T} e_{n}}{\left(h_{n}(c)\right)^{2}} \log \left(1+\frac{h_{n}(c)}{e_{n}} \sqrt{1-t}\right) \\
& \leq\left(c+\tau-\frac{4 \lambda_{2}^{T}}{h_{n}(c)}-c\right) \sqrt{1-t}+\frac{4 \lambda_{2}^{T} e_{n}}{\left(h_{n}(c)\right)^{2}} \log \left(1+\frac{h_{n}(c)}{e_{n}} \sqrt{1-t}\right) \\
& =\left(h_{n+1}(c)-c\right) \sqrt{1-t}+\varepsilon+\frac{4 \lambda_{2}^{T} e_{n}}{\left(h_{n}(c)\right)^{2}} \log \left(1+\frac{h_{n}(c)}{e_{n}} \sqrt{1-t}\right)-\varepsilon .
\end{aligned}
$$

As $h_{n}(c)>0$ and $e_{n}>0$ we conclude

$$
\begin{aligned}
x(1)-x(t) & \leq\left(h_{n+1}(c)-c\right) \sqrt{1-t}+\varepsilon+\frac{4 \lambda_{2}^{T} e_{n}}{\left(h_{n}(c)\right)^{2}} \log \left(1+\frac{h_{n}(c)}{e_{n}}\right)-\varepsilon \\
& =\left(h_{n+1}(c)-c\right) \sqrt{1-t}+e_{n+1}-\varepsilon .
\end{aligned}
$$

For $n=N$ we get

$$
x(1)-x(t) \leq e_{N}-\varepsilon+\left(h_{N}(c)-c\right) \sqrt{1-t} .
$$

On the other hand,

$$
x(1)-x(t)=x(1)-U_{2}(1)+U_{2}(1)-x(t) \geq-\varepsilon+U_{2}(1)-U_{2}(t) \geq-\varepsilon-c \sqrt{1-t} .
$$

Thus

$$
h_{N}(c) \sqrt{1-T}+e_{N} \geq 0 .
$$

Now we can send $\varepsilon \rightarrow 0$ and get with (3.5) $h_{N}(c) \geq 0$, a contradiction to (3.4).

Remark. Again, the statement is still true for any time $t>0$ other than 1 .

If we consider the backward Loewner equation with real initial values, i.e.,

$$
\dot{x}(t)=\frac{-2 \lambda_{1}(T-t)}{x(t)-U_{1}(T-t)}+\frac{-2 \lambda_{2}(T-t)}{x(t)-U_{2}(T-t)}, \quad x(0)=x_{0},
$$

then the solution may not exist for all $t \in[0, T]$. However, if two different solutions $x(t), y(t)$ with $x(0)=x_{0}>y_{0}=y(0)$ meet a singularity after some time, lets say $x(T)=y(T)=U_{2}(0)$, then $x_{0}$ and $y_{0}$ lie on different sides with respect to $U_{2}(T)$, i.e. 
$y_{0}<U_{2}(T)<x_{0}$. Consequently, there are are at most two initial values so that the corresponding solutions will meet in $U_{1}(0)$ or in $U_{2}(0)$, respectively.

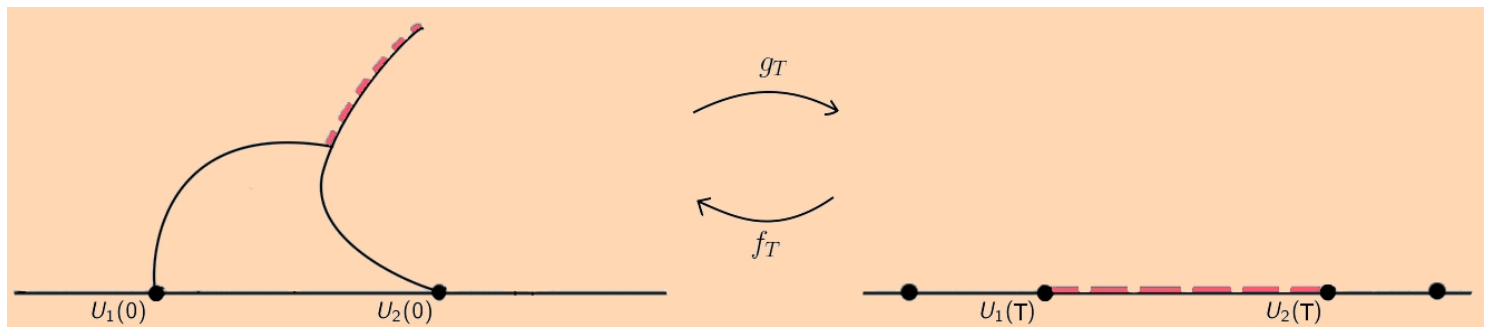

Figure 1. The left slit hits the right one at $t=T$.

The set of all real initial values whose solutions hit a singularity will be mapped by $f_{T}$ into $\mathbf{H}$. Figure 1 shows an example of a situation, where we have only one solution that hits $U_{1}(0)$, because all solutions with initial value in $\left(U_{1}(T), U_{2}(T)\right)$ will meet $U_{2}(t)$. So the whole interval $\left(U_{1}(T), U_{2}(T)\right)$ "belongs" to the second curve.

Now the hull $K_{1}$ consists of two disjoint curves, if and only if for each $s \in(0,1]$ and $j=1,2$ there are two different values $x_{0}, y_{0}$ such that the corresponding solutions of the backward equation (3.6) with $T=1$ satisfy $x(s)=y(s)=U_{j}(1-s)$. Another formulation varies the time $T$ and thus equation (3.6): $K_{1}$ consists of two disjoint curves, if and only if for each $T \in(0,1]$ and $j=1,2$ there are two different values $x_{0}, y_{0}$ with $x(T)=y(T)=U_{j}(0)$.

Proof of Theorem 1.2 (with $n=2$ ). Let $T \in(0,1]$. We have to show that for $j \in\{1,2\}$, there are exactly two real numbers $x_{0}$ and $y_{0}$ so that the solutions $x(t), y(t)$ of the backward equation (3.6) satisfy $x(T)=y(T)=U_{j}(0)$. We only consider $j=2$, the same arguments can be applied to $j=1$. First, we set $a_{n}:=U_{2}(0)-\frac{1}{n}$ for all $n \in \mathbf{N}$ such that $a_{n}>U_{1}(0)$. The solution of (3.2) with initial value $a_{n}$ exists up to time $T$ and doesn't hit $U_{2}(T)$ because of Lemma 3.3. Hence we can define $x_{n}:=g_{T}\left(a_{n}\right)$ and Lemma 3.4 implies that there is an $\varepsilon$ with $U_{2}(T)-x_{n}>\varepsilon$. The sequence $x_{n}$ is increasing and bounded above, and so it has a limit $x_{0}<U_{2}(T)$. Then the solution of (3.6) with $x_{0}$ as initial value satisfies $x(T)=\lim _{n \rightarrow \infty} a_{n}=U_{2}(0)$. The second value $y_{0}$ can be obtained in the same way by considering the sequence $U_{2}(0)+\frac{1}{n}$ instead of $a_{n}$.

\section{References}

[GS08] Gubiec, T., and P. Szymczak: Fingered growth in channel geometry: a Loewnerequation approach. - Phys. Rev. E (3) 77:4, 041602, 2008, 1-12.

[La05] LAWler, G.: Conformally invariant processes in the plane. - Math. Surveys Monogr. 114, 2005.

[Li05] Lind, J.: A sharp condition for the Löwner equation to generate slits. - Ann. Acad. Sci. Fenn. Math. 30:1, 2005, 143-158.

[LMR10] Lind, J., D. MARshall, and S. Rohde: Collisions and spirals of Loewner traces. - Duke Math. J. 154, 2010, 527-573.

[MR05] Marshall, D., and S. Rohde: The Loewner differential equation and slit mappings. J. Amer. Math. Soc. 18, 2005, 763-778.

[Pr93] Prokhorov, D. V.: Reachable set methods in extremal problems for univalent functions. - Saratov Univ., Saratov, 1993.

Received 9 May 2011

Revised received 14 October 2011 